\title{
Desenvolvimento e validação de metodologia para quantificação de alcalóides totais como berberina em fitoterápico contendo Berberis vulgaris $\mathrm{L}$.
}

\author{
Marcos A.C. Oliveira ${ }^{1}$, Miracy M. Albuquerque ${ }^{*}$, Haroudo S. Xavier ${ }^{2}$, Ruth R. Strattmann ${ }^{1}$, \\ Severino Grangeiro Júnior', Adelaide T. Queiroz ${ }^{3}$
}

\author{
${ }^{1}$ Núcleo de Controle de Qualidade de Medicamentos e Correlatos, Departamento de Ciências Farmacêuticas, \\ Universidade Federal de Pernambuco, 50740-521, Recife, PE, Brasil, \\ ${ }^{2}$ Laboratório de Farmacognosia, Departamento de Ciências Farmacêuticas, Universidade \\ Federal de Pernambuco, 50740-521, Recife, PE, Brasil, \\ ${ }^{3}$ Diniz \& Brandão S.A. Indústria e Comércio, Jaboatão dos Guararapes-PE. Praça Severina \\ Rita Coelho, 21/43, Cavaleiro, 54250-600, Jaboatão dos Guararapes-PE, Brasil
}

\begin{abstract}
RESUMO: A Robusterina ${ }^{\circledR}$ é um produto fitoterápico, com eficácia no tratamento de disfunções do ciclo menstrual. Em sua composição encontram-se Berberis vulgaris L., de ação sedativa e antiespasmódica; Gossypium herbaceum L., enemagoga, hemostática e ocitócica; Viburnum opulus L., antiespasmódico nas cólicas menstruais. De acordo com a Resolução RDC N 48, de 16 de março de 2004, observa-se que o produto adequa-se na definição de Fitoterápico. A presença de alcalóides em Berberis vulgaris e a ausência de metodologias analíticas de quantificação para o produto, nos incentivaram a propor e validar um método apoiando-nos na Resolução RE $\mathrm{N}^{\circ} 899$, de 29 de Maio de 2003. Tal metodologia fundamenta-se na determinação espectrofotométrica de alcalóides utilizando-se Dragendorff como reagente precipitante, e o sulfato de berberina Merck ${ }^{\circledR}$, como substância química de referência. A curva de calibração foi determinada com seis concentrações entre 40 e $200 \mu \mathrm{g} / \mathrm{mL}$. A equação da reta é y $=0,0038 \mathrm{x}+0,0092$ com $\mathrm{R}^{2}$ de 0,9996 . Os parâmetros robustez, precisão, especificidade, limite de detecção e quantificação e exatidão foram avaliados estatisticamente com intervalo de confiança de 95\% (teste t de Student, ANOVA).
\end{abstract}

Unitermos: Robusterina ${ }^{\circledR}$, fitoterápico, validação, alcalóides, doseamento.

\begin{abstract}
Development and validation of a method for the quantification of total alkaloids as berberine in an herbal medicine containing Berberis vulgaris L.”. Robusterina ${ }^{\circledR}$ is a herbal medicine, with effectiveness in the treatment of menstrual cycle disfunctions. Its composition includes Berberis vulgaris L., with sedative and antispasmodic action; Gossypium herbaceum L., with emmenagogue, hemostatic and ocitocic action; Viburnum opulus L., with antispasmodic action for the treatment of menstrual colics. In accordance with Resolution RDC $\mathrm{N}^{\circ} 48$, of 16 of March of 2004 (ANVISA, Brazil) the product meets the definition of "Fitoterápico" (phytotherapeutic agent). The presence of alkaloids in Berberis vulgaris and the absence of analytical methodologies for quantification of the product, stimulated us to develop and validate a method in accordance with Resolution RE N ${ }^{\circ} 899$, of 29 of May of 2003. Such methodology is based on the determination of alkaloids using a spectrophotometric method, with Dragendorff as a precipitating reagent, and using berberine sulphate, as a standard. The calibration curve was determined with six concentrations ranging between 40 and $200 \mu \mathrm{g} / \mathrm{mL}$. The equation of the calibration curve is $\mathrm{y}=0.0038 \mathrm{x}+$ 0.0092 with $\mathrm{R}^{2}$ of 0.9996 . The parameters robustness, precision, specificity, limit of detection and quantification and accuracy have been evaluated using $95 \%$ confidence interval (test $t$ of Student, ANOVA).
\end{abstract}

Keywords: Robusterina ${ }^{\circledR}$, herbal medicine, validation, alkaloids, quantification.

\section{INTRODUÇÃO}

A Robusterina ${ }^{\circledR}$ é um medicamento (solução oral) de origem exclusivamente vegetal. Encontra-se no mercado nacional desde 1932, sendo utilizada no tratamento das disfunções e regulação do ciclo menstrual e, por estimular a contração uterina, seu uso é contraindicado no período gestacional. A dose média diária é de uma colher de sopa $(15 \mathrm{~mL}) 3$ vezes ao dia. $\mathrm{O}$ tratamento deve ser suspenso quando houver normalização do ciclo menstrual, ou no caso de gravidez. Apresenta-se em sua constituição três plantas: Berberis vulgaris, Gossypium herbaceum e Viburnum opulus.

Para o estudo, foi dada ênfase a Berberis vulgaris, pois é a única planta que apresenta na sua constituição alcalóides, para a qual foi desenvolvida 
e validada uma metodologia analítica baseada na determinação espectrofotométrica de alcalóides, utilizando Dragendorff como reagente precipitante.

Berberis vulgaris, é uma Berberidaceae arbustiva com uma altura de 2 a 3 metros (Alonso, 1998). É nativa da Europa e Ásia Oriental, e, na atualidade, encontrada também no Norte da África e partes da América e Ásia Central (PDR for Herbal Medicines, 2000).

Apresentando uma vasta sinonímia vulgar, é conhecida como agracejo (espanhol) (Bruneton, 1991), barberry (inglês), bérberis (português), crespino (italiano) (Alonso, 1998) e épine-vinette (francês) (Duke, 1985). Apresenta outros nomes como berberry, pipperidge, jaundice berry, sow berry, mountain grape e oregon grape (PDR for Herbal Medicines, 2000). As partes da planta utilizadas para uso medicinal são os frutos, a casca da raiz e as folhas (Alonso, 1998).

Os frutos têm em sua constituição dextrose, levulose, ácido cítrico, ácido tartárico, goma e pectina (Alonso, 1998). Na casca das raízes são encontrados alcalóides como a berberina, palmatina, columbamina, berbamina, oxyacantina e magnoflorina perfazendo um total de 3\% em alcalóides, o que confere ao córtex uma coloração amarela. A atividade farmacológica da planta é atribuída aos alcalóides presentes, principalmente a berberina (Pizzorno; Murray, 1985). A berberina está presente em outras famílias de plantas (Papaveraceae, Ranunculaceae, etc) (Alonso, 1998). Também são encontrados ácidos málico, cítrico, chelidonico e tartárico, além de resina e taninos (Duke, 1985).

Em concentrações normais (até $500 \mathrm{mg}$ ) (http:// www.fredmeyer.com/Es-Herb/Barberry.htm), a berberina é um alcalóide com propriedades digestivas, eupépticas (carminativas), orexígenas (Alonso, 1998), febrífuga e, externamente, é utilizado como analgésico em dores provocadas pela úlcera (Duke, 1985). São também atribuídos a berberina, ações antisséptica e ocitócica (Cunha et. al., 2003) e uma atividade uterina (Farnsworth, 1975), antiinflamatória (Barbosa-Filho et al., 2006a) e inibidor da enzima acetilcolinesterase (Barbosa-Filho et al., 2006b). Possui ação antimicrobiana (Pizzorno; Murray, 1985). Mostrou-se eficaz no tratamento de diarréias causadas por enterotoxinas, como Vibrio cholerae e Escherichia coli (Preininger, 1975). Tradicionalmente a raiz é usada para o tratamento de litíase biliar (Bruneton, 1991). O sulfato de berberina tem apresentado atividades frente a tumores tipo B1, RB e PS (Fernald, 1958).

A Resolução RDC $\mathrm{N}^{\circ} 48$, de 16 de Março de 2004, da Agência Nacional de Vigilância Sanitária (ANVISA, 2004), regula o registro dos medicamentos fitoterápicos e, exige relatório de controle de qualidade, incluindo então as metodologias analíticas. A falta de metodologia analítica para a Berberis vulgaris, levou-nos a desenvolver uma metodologia analítica para o produto, a qual foi validada, de acordo com a Resolução RE $N^{\circ}$ 899, de 29 de Maio de 2003, do Ministério da Saúde (ANVISA,
2003). De acordo com esta resolução, a metodologia será considerada validada, desde que sejam avaliados parâmetros relacionados: Especificidade/Seletividade, Curva de Calibração/Linearidade, Intervalos de Curva de Calibração, Precisão, Limite de Detecção (LD), Limite de Quantificação (LQ), Exatidão e Robustez.

A validação da metodologia analítica é de grande importância para a Garantia da Qualidade Analítica e se constitui numa das exigências das normas de Boas Práticas de Fabricação (BPF) vigentes. Os métodos de ensaio para avaliar a conformidade dos produtos farmacêuticos com especificações estabelecidas devem atingir padrões adequados de exatidão, precisão e confiabilidade (Barros, 2002; Ribeiro et al., 2005; Bara et al., 2006; Martins; Brandão, 2006).

A metodologia analítica proposta e validada foi desenvolvida a partir de pesquisas, tendo-se como destaque o trabalho desenvolvido por Sreevidya e Mehrotra (2003).

\section{MATERIAL E MÉTODOS}

O material utilizado (Robusterina ${ }^{\circledR}$, tintura de berberis, tintura de viburno e tintura de algodoeiro) foi gentilmente cedido pelo Laboratório Diniz \& Brandão.

\section{Material}

Utilizaram-se vidrarias certificadas por lote, do fabricante Thermex ${ }^{\circledR}$, espectrofotômetro Varian Modelo 6345, balança analítica Mettler Modelo AE 260 e centrifuga Fanem Modelo Série 204N/116653.

A substância química de referência (S.Q.R) determinada para a validação da metodologia analítica foi o sulfato de berberina, Merck ${ }^{\circledR}$. No experimento foram manuseadas as seguintes substâncias: ácido clorídrico diluído ( $\mathrm{HCl} 1 \mathrm{~N})$; Reagente de Dragendorff; nitrato de bismuto pentahidratado, (VETEC); iodeto de potássio (Mallinckrodt); água destilada; ácido acético glacial; álcool etílico absoluto (J.T. Baker); álcool etílico absoluto (Nuclear); sulfito de sódio $\left(\right.$ Merck $\left.^{\circledR}\right)$; ácido nítrico concentrado; tiouréia $\left(\right.$ Merck $\left.^{\circledR}\right)$; Robusterina ${ }^{\circledR}$ (Laboratório Diniz \& Brandão, Lote 0405033 validade 05/2007).

\section{Preparação da solução de sulfato de berberina S.Q.R.}

Foram pesados $61,3 \mathrm{mg}$ de sulfato de berberina (S.Q.R) (equivalente a $50 \mathrm{mg}$ de berberina) e transferidos quantitativamente para balão volumétrico de $50 \mathrm{~mL}$ e completou-se o volume com água destilada, e, em seguida transferiu-se $5 \mathrm{~mL}$ de cada solução (triplicata) e acidificou-se a pH entre 2-2,5 com $\mathrm{HCl} 1 \mathrm{~N}$; transferiu-se $5 \mathrm{~mL}$ da solução acidificada para cada tubo de centrífuga (triplicata) e a cada tubo foram adicionados $2 \mathrm{~mL}$ do Reagente de Dragendorff e centrifugou-se a $2400 \mathrm{rpm} /$ 30 minutos; desprezou-se o sobrenadante e tratou-se

Rev. Bras. Farmacogn. Braz J. Pharmacogn 16(3):jul/set. 2006 
o resíduo com $1 \mathrm{~mL}$ de álcool etílico absoluto; foram adicionados $2 \mathrm{~mL}$ de sulfito de sódio a $1 \%$ e centrifugouse a $2400 \mathrm{rpm} / 30$ minutos e a seguir desprezou-se o sobrenadante e tratou-se o resíduo com $2 \mathrm{~mL}$ de ácido nítrico concentrado; transferiu-se o conteúdo resultante para balão volumétrico de $50 \mathrm{~mL}$, completou-se o volume com água destilada; foi tomado $1 \mathrm{~mL}$ desta solução e adicionou-se $5 \mathrm{~mL}$ de tiouréia a $3 \%(\mathrm{p} / \mathrm{V})$; a mistura de ácido nítrico e tiouréia foi usada como branco; para a amostra foi procedida a leitura a $435 \mathrm{~nm}$.

A linearidade foi obtida entre 40,0 e $200,0 \mu \mathrm{g} /$ $\mathrm{mL}$

\section{Preparação da amostra}

Foram tomados $40 \mathrm{~mL}$ do produto (que corresponde a uma concentração $6,66 \mathrm{mg} / \mathrm{mL}^{*}$ de Berberis vulgaris na Robusterina ${ }^{\circledR}$ ) e acidificou-se a $\mathrm{pH}$ entre 2-2,5 com $\mathrm{HCl} 1 \mathrm{~N}$; distribuiu-se este volume em 4 tubos de centrífuga; a cada tubo adicionou-se $4 \mathrm{~mL}$ do Reagente de Dragendorff e foram centrifugados a 2400 rpm / 30 minutos; prosseguiu-se conforme descrito na solução de sulfato de berberina S.Q.R e as amostras foram lidas em espectrofotômetro a $435 \mathrm{~nm}$, utilizando a mistura de ácido nítrico e tiouréia como branco, de acordo com o descrito por Sreevidya e Mehrotra (2003).

*A concentração de $6,66 \mathrm{mg} / \mathrm{mL}$ é resultante da produção da Robusterina ${ }^{\circledR}$, onde é adicionado $1 \mathrm{~kg}$ de material vegetal (casca de raiz) de Berberis vulgaris para um volume final de $150 \mathrm{~L}$ do produto.

\section{Estudo da validação}

Os parâmetros avaliados foram a robustez, linearidade, especificidade, precisão, limite de detecção (LD), limite de quantificação (LQ), exatidão.

Robustez: A robustez do método foi determinada sobre as variações de $\mathrm{pH}$, velocidade de centrifugação e marca de solvente. As análises foram realizadas em quadruplicata com as amostras reais do fitoterápico Robusterina ${ }^{\circledR}$. A sua avaliação foi feita através da análise de variância (ANOVA) one-way.

Linearidade: A linearidade foi avaliada através da análise de regressão linear pelo método dos mínimos quadrados dos pontos médios de 3 (três) curvas de calibração autênticas, usando-se seis concentrações $(40$,

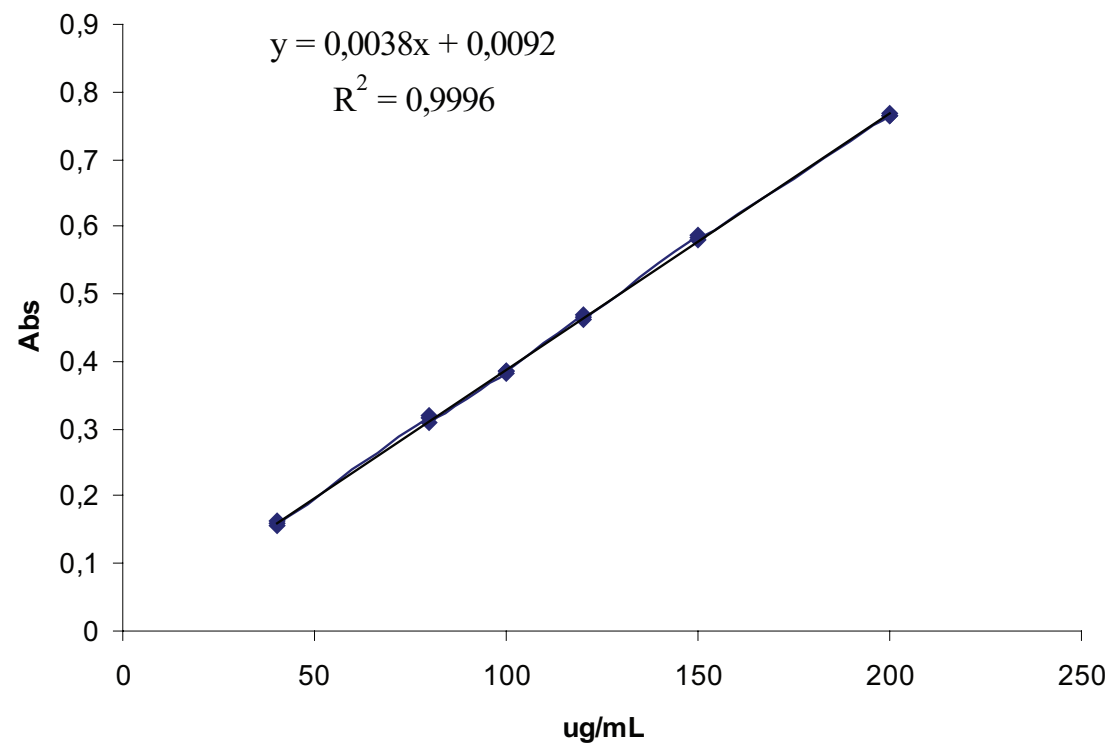

Figura 1. Curva de regressão linear obtida da média das três curvas de calibração autênticas

Tabela 1. Resultados da linearidade - 3 curvas autênticas do sulfato de berberina

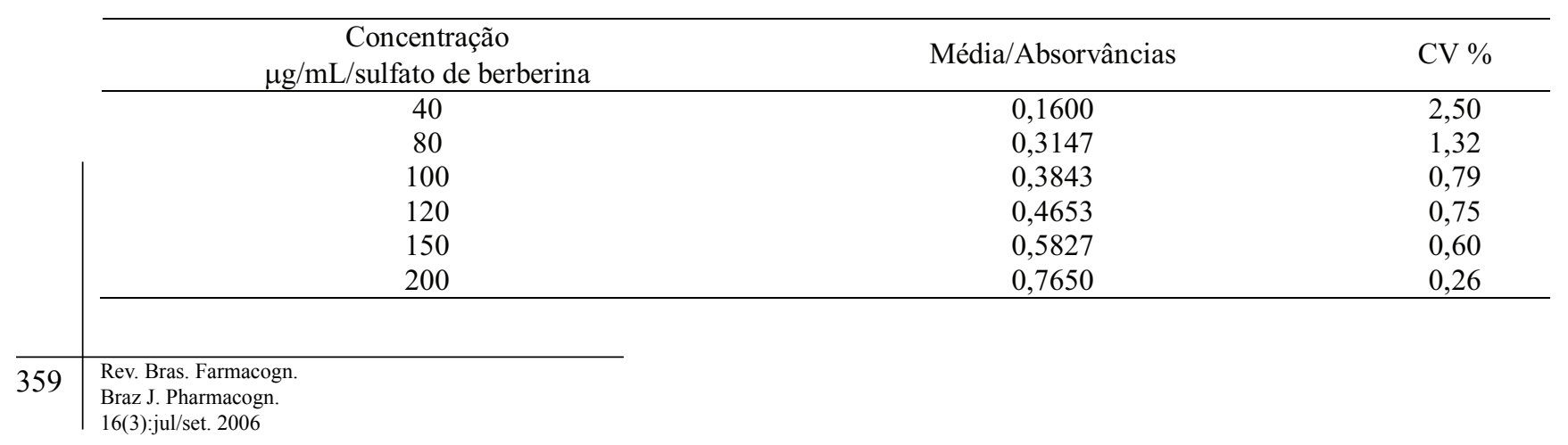


Tabela 2. Resultados da análise de variância para linearidade

\begin{tabular}{l|l|l|l|l|l}
\hline Fonte & SQ & gl & MQ & F & F-crítico \\
\hline Modelo SQ reg & 0,670816935 & 1 & 0,670816935 & 36130,33 & 4,4940 \\
\hline Residual SQ res & 0,000297065 & 16 & $1,85666 \mathrm{E}-05$ & \multicolumn{2}{|l}{ Curva Linear* } \\
\hline Falta de ajuste SQ faj & 0,000154399 & 4 & $3,85997 \mathrm{E}-05$ & 3,24 & 3,25 \\
\hline Erro puro SQ erp & 0,000142667 & 12 & $1,18889 \mathrm{E}-05$ & \multicolumn{2}{|l}{ Não há falta de ajuste** } \\
\cline { 1 - 3 } Total SQ tot & 0,671114 & 17 & 0,039477294 & & \\
\hline
\end{tabular}

$\mathrm{SQ}=$ Soma Quadrática; $\mathrm{gl}=$ Graus de Liberdade; $\mathrm{MQ}=$ Média Quadrática

* Regressão Estatisticamente Significativa

** Proporcionando uma curva linear

Tabela 3. Avaliação da robustez variando o parâmetro de $\mathrm{pH}$

\begin{tabular}{lcccc}
\hline \multirow{2}{*}{$\mathrm{pH}$} & Amostra 1 & Amostra 2 & Amostra 3 & Amostra 4 \\
\cline { 2 - 5 } & $\mu \mathrm{g} / \mathrm{mL}$ & $\mu \mathrm{g} / \mathrm{mL}$ & $\mu \mathrm{g} / \mathrm{mL}$ & $\mu \mathrm{g} / \mathrm{mL}$ \\
\hline 2,3 & 29,20 & 29,20 & 29,20 & 28,32 \\
2,4 & 28,48 & 29,00 & 29,20 & 28,84 \\
2,5 & 28,68 & 29,00 & 28,68 & 28,32 \\
Média & 28,79 & 29,07 & 29,03 & 28,49 \\
Desv Pad & 0,3717 & 0,1155 & 0,3002 & 0,3002 \\
CV $(\%)$ & 1,2911 & 0,3973 & 1,0343 & 1,0537 \\
\hline
\end{tabular}

Tabela 4. Avaliação da robustez variando o pH de acordo com a ANOVA.

\begin{tabular}{l|c|c|c|c|c|c}
\hline Fonte de variação & SQ & gl & MQ & F & valor-P & F crítico \\
\hline Entre grupos & 0,20 & 2 & 0,10 & 0,826 & 0,468 & 4,256 \\
Dentro dos grupos & 1,09 & 9 & 0,12 & & & \\
Total & 1,29 & 11 & & & & \\
\hline
\end{tabular}

Tabela 5. Avaliação de robustez variando o parâmetro de velocidade de centrifugação.

\begin{tabular}{lcccc}
\hline \multirow{2}{*}{$\mathrm{VC}^{*}(\mathrm{rpm})$} & Amostra 1 & Amostra 2 & Amostra 3 & Amostra 4 \\
\cline { 2 - 5 } & $\mu \mathrm{g} / \mathrm{mL}$ & $\mu \mathrm{g} / \mathrm{mL}$ & $\mu \mathrm{g} / \mathrm{mL}$ & $\mu \mathrm{g} / \mathrm{mL}$ \\
\hline 2000 & 29,90 & 29,90 & 30,04 & 29,90 \\
2200 & 30,04 & 30,02 & 29,90 & 29,90 \\
2400 & 29,90 & 29,82 & 29,90 & 29,90 \\
Média & 29,95 & 29,91 & 29,95 & 29,90 \\
Desv Pad & 0,0808 & 0,1007 & 0,0808 & 0,000 \\
CV $(\%)$ & 0,2699 & 0,3365 & 0,2699 & 0,000 \\
\hline
\end{tabular}

* Velocidade de Centrifugação

Tabela 6. Avaliação da robustez variando a velocidade de centrifugação de acordo com a ANOVA.

\begin{tabular}{lcccccc}
\hline Fonte de variação & SQ & gl & MQ & F & valor-P & F crítico \\
\hline Entre grupos & 0,01 & 2 & 0,005 & 1,827 & 0,215 & 4,256 \\
Dentro dos grupos & 0,04 & 9 & 0,004 & & & \\
Total & 0,05 & 11 & & & & \\
\hline
\end{tabular}


$80,100,120,150$ e $200 \mu \mathrm{g} / \mathrm{mL})$. Para verificação da significância da equação de regressão, foram efetuados testes de ajuste do modelo linear e validade da regressão. Para determinação da linearidade foi utilizado o sulfato de berberina S.Q.R.

Especificidade: Foi realizada uma mistura das tinturas de algodoeiro e de viburno e procedeu-se conforme descrito para S.Q.R. A mistura funcionou como placebo para provar a ausência de alcalóides nestas duas plantas (Gossypium herbaceum e Viburnum opulus).

Precisão: A repetibilidade foi determinada pela análise de seis amostras individuais do fitoterápico Robusterina $^{\circledR}$. A precisão intermediária foi determinada em dois dias por dois analistas diferentes, utilizando também amostras da Robusterina ${ }^{\circledR}$.

A repetibilidade foi expressa através do coeficiente de variação $(\mathrm{CV})$ e a precisão intermediária foi expressa, além do $\mathrm{CV}$, através do intervalo de confiança da média pelo teste $\mathrm{t}$ de Student (Ribani et al., 2004).

Limite de detecção (LD): É calculado também na prática como uma concentração que produz um sinal três vezes maior que o nível de ruído médio medido com o branco ou solução controle (Leite, 1989).

$$
\mathrm{LD}=\frac{3 \mathrm{DP}}{\alpha}
$$

$\mathrm{DP}=$ Desvio Padrão Médio da menor concentração $\alpha=$ Médias dos coeficientes angulares

Limite de Quantificação (LQ): Para se realizar o LD e LQ foi considerada o desvio padrão da reta com relação à absorvância do primeiro nível de concentração $(40 \mu \mathrm{g} / \mathrm{mL})$ do sulfato de berberina nas três curvas de calibração e seus coeficiente angulares (inclinação da reta). Foi considerada a razão de três vezes da linha da base para o LD e dez vezes para o LQ.

$$
\mathrm{LQ}=\frac{10 \mathrm{DP}}{\alpha}
$$

$\mathrm{DP}=$ Desvio Padrão Médio da menor concentração

$\alpha=$ Médias dos coeficientes angulares

Exatidão: A exatidão do método foi avaliada em três níveis de concentração: 50, 100 e 150\%, onde $100 \%$ correspondeu a concentração de $29,46 \mu \mathrm{g} / \mathrm{mL}$, determinada a partir da média da análise de seis amostras de Robusterina ${ }^{\circledR}$ no parâmetro da repetibilidade. Antes de obter a exatidão do método foi determinada uma aplicação do método de adição do padrão (sulfato de berberina), para que obtivéssemos um valor nominal na amostra real do fitoterápico Robusterina ${ }^{\circledR}$. Após detecção do aumento do sinal do analito provocado pela adição do padrão, por diferença foi obtida a concentração de analito originalmente presente no volume da matriz. Após certificação deste processo, foram preparadas as amostras do fitoterápico Robusterina ${ }^{\circledR}$, conforme descrito para a preparação da S.Q.R. sendo que, para a quantidade de $50 \%$, tomou-se o volume de $0,5 \mathrm{~mL}$, para $100 \%$, volume de $1,0 \mathrm{~mL}$ e para $150 \%$, um volume de $1,5 \mathrm{~mL}$, que foram adicionados com $5 \mathrm{~mL}$ de tiouréia e lidos em espectrofotômetro a $435 \mathrm{~nm}$.

Os testes foram feitos em triplicata de cada nível de concentração e foram avaliados através do teste $t$ de Student, comparando-se os resultados em relação ao valor teórico definido para cada concentração analisada.

\section{RESULTADOS E DISCUSSÃO}

Foi feita uma varredura espectrofotométrica com o sulfato de berberina, na concentração de $100 \mu \mathrm{g} /$ $\mathrm{mL}$, para determinar o comprimento de onda de $435 \mathrm{~nm}$, de acordo com Sreevidya e Mehrotra (2003). A partir desta observação, iniciou-se a determinação da curva de calibração tomando os dados descritos na Tabela 1.

\section{Linearidade}

Os resultados foram plotados e está representado em gráfico na figura 1 , tendo no eixo dos $\mathrm{x}$ a concentração de sulfato de berberina e no eixo dos y absorvância. Pelo método dos mínimos quadrados obteve-se a equação da reta $y=0,0038 x+0,0092$. A análise de regressão linear demonstrou um coeficiente de correlação $\mathrm{R}^{2}=0,9996$ valor que comprova a linearidade do método (Figura 1).

A partir da análise de variância (Tabela 2) podemos testar a validação do modelo e a significância estatística da curva ajustada. As análises de variância dos dados demonstraram que o método é linear, na faixa de concentração testada $(40-200 \mu \mathrm{g} / \mathrm{mL})$ e que não há falta de ajuste do modelo, já que esta relação apresentou um valor de $\mathrm{F}_{\text {calculado }}=3,24$, abaixo do valor crítico $\mathrm{F}_{\text {tabelado }}=$ 3,25 com 4 e 12 graus de liberdade e $95 \%$ de confiança mostrando que o modelo linear está bem ajustado na faixa de concentração estudada (Pimentel; Barros, 1996).

\section{Robustez}

Foram analisadas amostras em quadruplicata para cada parâmetro de variação estudado: alteração de pH $(2,3 ; 2,4$ e 2,5), velocidade de rotação $(2000,2200$ e $2400 \mathrm{rpm}$ ) e marca do solvente (álcool etílico absoluto).

\section{Avaliação da robustez variando o pH}

A Tabela 3, mostra a avaliação da robustez, variando o parâmetro de $\mathrm{pH}$.

Para o parâmetro avaliado $(\mathrm{pH})$, verificou-se estatisticamente através do ANOVA (análise de variância) que não há diferença significativa entre os resultados. Obtendo-se um $\mathrm{F}_{\text {calculado }}=0,826$ menor que o $\mathrm{F}_{\text {tabelado }}=$ 4,256, o método apresenta-se robusto, não existindo, em um nível de $95 \%$ de confiança, variação estatisticamente significativa quanto ao $\mathrm{pH}$ (Tabela 4).

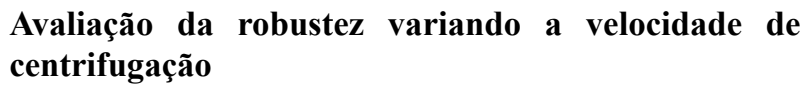


Tabela 7. Avaliação da robustez variando o parâmetro Marca do Solvente.

\begin{tabular}{lcccc}
\hline \multirow{2}{*}{ Marca do Solvente } & Amostra 1 & Amostra 2 & Amostra 3 & Amostra 4 \\
\cline { 2 - 5 } & $\mu \mathrm{g} / \mathrm{mL}$ & $\mu \mathrm{g} / \mathrm{mL}$ & $\mu \mathrm{g} / \mathrm{mL}$ & $\mu \mathrm{g} / \mathrm{mL}$ \\
\hline Nuclear & 29,36 & 29,52 & 29,74 & 29,74 \\
J. T. Backer & 29,00 & 29,20 & 29,20 & 29,90 \\
Média & 29,18 & 29,36 & 29,47 & 29,82 \\
CV $(\%)$ & 0,8724 & 0,7707 & 1,2957 & 0,3794 \\
\hline
\end{tabular}

Tabela 8. Teste-t - robustez de marca do solvente.

\begin{tabular}{l|c|c}
\hline & Nuclear & J. T. Backer \\
\hline Média & 0,0342 & 29,32 \\
Variância & 1,2156 & 0,1558 \\
\cline { 2 - 4 } Stat $\mathrm{t}$ & 1,2156 & \\
$\mathrm{P}(\mathrm{T}<=\mathrm{t})$ bi-caudal & 0,2698 & \\
$\mathrm{~T}$ crítico bi-caudal & 2,4469 & \\
\hline
\end{tabular}

Tabela 9. Resultados da repetibilidade.

\begin{tabular}{lcc}
\hline Amostra/Média & $\mu \mathrm{g} / \mathrm{mL}$ & Concentração \% \\
\hline Média & 29,46 & 0,4420 \\
Desv Pad & 0,5147 & 0,0077 \\
CV\% & 1,7468 & 1,7468 \\
\hline
\end{tabular}

Tabela 10. Resultados da precisão intermediária, de 4 amostras tratadas em dias consecutivos e analistas distintos

\begin{tabular}{lcccc}
\hline Dias/Analistas & Amostra-1 & Amostra-2 & Amostra-3 & Amostra-4 \\
\hline Média & 28,19 & 28,58 & 28,71 & 28,45 \\
Desv Pad & 0,9940 & 0,5200 & 0,2600 & 0,6609 \\
CV\% & 3,5268 & 1,8195 & 0,9056 & 2,3237 \\
\hline
\end{tabular}

Tabela 11. Precisão Intermediária de acordo com a ANOVA.

\begin{tabular}{lccccc}
\hline Fonte de variação & SQ & gl & MQ & F calculado & F tabelado \\
\hline Analistas & 0,60 & 3 & 0,20 & 0,38 & 3,86 \\
Dias & 0,47 & 3 & 0,16 & 0,29 & 3,86 \\
Erro & 4,82 & 9 & 0,53 & & \\
Total & 5,89 & 15 & & & \\
\hline
\end{tabular}

Tabela 12. Resultados da Exatidão para concentrações baixa, média e alta.

\begin{tabular}{lccc}
\hline Determinações & $13,50 \mu \mathrm{g} / \mathrm{mL}$ & $29,46 \mu \mathrm{g} / \mathrm{mL}$ & $45,47 \mu \mathrm{g} / \mathrm{mL}$ \\
\hline Média & 13,75 & 29,28 & 45,41 \\
Desv Pad & 0,1791 & 0,1064 & 0,2127 \\
CV $(\%)$ & 1,3023 & 0,3632 & 0,4684 \\
\hline
\end{tabular}

Tabela 13. Teste $t$ de Student para exatidão - Concentrações baixa (50\%), média (100\%)e alta (150\%).

\begin{tabular}{lcc}
\hline Percentual & T calculado & T tabelado \\
\hline $50 \%$ & 2,4173 & 4,3030 \\
$100 \%$ & 2,9314 & \\
$150 \%$ & 0,4885 & \\
\hline
\end{tabular}


A Tabela 5 mostra os resultados da robustez variando o parâmetro de velocidade de centrifugação.

Para o parâmetro avaliado (velocidade de centrifugação), verificou-se estatisticamente através do ANOVA (análise de variância) que não há diferença significativa entre os resultados. Obtendo-se um $\mathrm{F}$ $=1,827$ menor que o $\mathrm{F}_{\text {tabelado }}=4,256$, o método apresentase robusto, não existindo, em um nível de $95 \%$ de confiança, variação estatisticamente significativa quanto à velocidade de centrifugação (Tabela 6).

\section{Avaliação da robustez variando a marca do solvente}

O álcool etílico é utilizado para lavar o resíduo antes de se adicionar o sulfito de sódio, conforme descrito para a preparação da S.Q.R. sulfato de berberina e amostra.

Os resultados estão na Tabela 7.

Para a avaliação da robustez variando a marca do solvente álcool etílico absoluto, utilizou-se o teste t de Student como ferramenta estatística para comparar as médias entre eles. Sendo o $\mathrm{T}_{\text {calculado }}(1,2156)$ menor que o $\mathrm{T}_{\text {tabelado }}(2,4469)$ não há diferença estatisticamente significativa com um nível de $95 \%$ de confiança entre a média da concentração do solvente álcool etílico absoluto da marca Nuclear e o da marca J.T. Backer. Os resultados afirmam que o método é robusto para o parâmetro estudado (Tabela 8).

\section{Repetibilidade}

A Tabela 9 explicita os resultados do ensaio de repetibilidade.

Observando-se os resultados, podemos concluir que o método tem uma boa repetibilidade, visto que o coeficiente de variação (CV\%) é inferior ao especificado pela resolução vigente, que é de $5 \%$.

\section{Precisão intermediária}

A precisão intermediária foi realizada em diferentes dias e com diferentes analistas, utilizando a mesma concentração. A Tabela 10 mostra os resultados da precisão intermediária, entre dias e analistas.

O estudo da precisão do método entre ensaios demonstrou que não há grandes diferenças entre as amostras analisadas individualmente em pequeno intervalo de tempo, assim como a precisão entre dias e entre analistas e os resultados encontrados demonstram que estas se enquadram dentro dos limites especificados. $\mathrm{Na}$ análise de variância o $\mathrm{F}_{\text {calculado }}$ foi menor que o $\mathrm{F}_{\text {tabelado }}$ não havendo diferença estatisticamente significativa em um nível de $95 \%$ de confiança entre os dois analistas (Tabela 11).

Limite de detecção (LD) e limite de quantificação (LQ)
A partir do momento que se define que o método é linear, podem ser calculados os valores de LD e LQ, utilizando a média do desvio padrão (DP) do primeiro ponto da reta e coeficientes angulares (ic). O LD calculado foi de $3,1578 \mu \mathrm{g} / \mathrm{mL}$ e o LQ foi de $10,5263 \mu \mathrm{g} / \mathrm{mL}$.

\section{Especificidade}

Foi preparada uma mistura com as tinturas de algodoeiro e de viburno na proporção 1:1, que também fazem parte da tintura de Robusterina, e que não apresentam alcalóides. A análise foi determinada em quadruplicata, para testar a especificidade do método para alcalóides. O método mostrou-se específico para alcalóides, pois as leituras de absorvância foram próximas a zero.

\section{Exatidão}

A determinação da exatidão foi realizada no mesmo dia com três concentrações diferentes baixa, média e alta $(50 \%(13,50 \mu \mathrm{g} / \mathrm{mL}) ; 100 \%(29,46 \mu \mathrm{g} / \mathrm{mL})$; $150 \%(45,47 \mu \mathrm{g} / \mathrm{mL}))$.

A exatidão do método foi comprovada pelo estudo de três concentrações diferentes $(50 \%$, $100 \%, 150 \%$ ) e o percentual de recuperação nas três concentrações analisadas encontrou-se dentro dos limites como mostram os resultados da Tabela 12.

O estudo estatístico aplicado foi o t de Student o qual demonstrou que o $\mathrm{T}_{\text {calculado }}$ foi menor que o $\mathrm{T}_{\text {tabelado }}$ comprovando que não houve diferença significativa entre as médias das três concentrações analisadas com um intervalo de 95\% de confiança (Tabela 13).

Conforme dito anteriormente, o trabalho executado por Sreevidya e Mehrotra (2003), foi de grande valia para o artigo, pois a metodologia sugerida por eles nos forneceu base suficiente para desenvolver e validar uma metodologia analítica para o produto Robusterina ${ }^{\circledR}$ .Em tal artigo os autores utilizaram a Berberis aristata e outras plantas (Solanum nigrum e Piper longum) que apresentam alcalóides para mostrar que o método responde no que diz respeito de doseamento de alcalóides. Como a Robusterina ${ }^{\circledR}$ apresenta casca de raiz de Berberis vulgaris $L$., proporcionou-se a oportunidade de dosear alcalóides totais (como berberina), no produto, trazendo um subsídio de controle de qualidade para a empresa fornecedora, para apresentar para os órgãos reguladores (ANVISA). Este método pode ser aplicado numa análise de rotina de uma indústria de medicamentos fitoterápicos pela sua facilidade de execução.

\section{AGRADECIMENTOS}

Agradecemos à CAPES pela concessão bolsa de estudo ao primeiro autor, ao Laboratório Diniz \& Brandão (em nome da Farmacêutica Adelaide Tavares) pelo fornecimento das tinturas e da Robusterina ${ }^{\circledR}$ para o desenvolvimento do trabalho, para a coordenadora 
do Núcleo de Controle de Qualidade de Medicamentos e Correlatos profa. Miracy e sua equipe e para o prof. Haroudo Satiro Xavier do Laboratório de Farmacognosia.

\section{REFERÊNCIAS}

Alonso JR 1998. Tratado de Fitomedicina: bases clínicas y farmacológicas. Isis Ediciones SRL, p.175-176.

ANVISA 2003. Agência Nacional de Vigilância Sanitária, Resolução RE N 899, de 29 de Maio de 2003. Diário Oficial da República Federativa do Brasil, Brasília DOU de 02/06/2003.

ANVISA 2004. Agência Nacional de Vigilância Sanitária, Resolução RDC N48, de 16 de Março de 2004. Diário Oficial da República Federativa do Brasil, Brasília DOU de 18/03/2004.

Bara MTF, Ribeiro PAM, Arantes MCB, Amorim LLSS, Paula JR 2006. Determinação do teor de princípios ativos em matérias-primas vegetais. Rev Bras Farmacogn 16: 211-215.

Barbosa-Filho JM, Piuvezam MR, Moura MD, Silva MS, Lima KVB, Cunha EVL, Fechine IM, Takemura OS 2006a. Anti-inflammatory activity of alkaloids: A twenty-century review. Rev Bras Farmacogn 16: 109-139.

Barbosa-Filho JM, Medeiros KCP, Diniz MFFM, Batista LM, Athayde-Filho PF, Silva MS, Cunha EVL, Almeida JRGS, Quintans-Júnior LJ 2006b. Natural products inhibitors of the enzyme acetylcholinesterase. Rev Bras Farmacogn 16: 258-285.

Barros CB 2002. Validação de métodos analíticos. Biológico 64: $175-177$.

Bruneton J 1991. Elementos de Fitoquimica y de Farmacognosia. Zaragoza: Editorial ACRIBIA.

Cunha AP, Silva AP, Roque OR 2003. Plantas e Produtos Vegetais em Fitoterapia. Lisboa: Fundação Calouste Gulbenkian.

Duke JA 1985. CRC Handbook of Medicinal Herbs. Florida: CRC Press, Inc, p. 78.

Farnsworth N 1975. Potential value of plants as sources of a new antifertility agents. J Pharm Sci 64: 535-593.

Fernald ML, Kinsey AC, Rollins RC 1958. Edible wilds plants of Eastern North America. New York: Harper and Brothers.

http:/www. fredmeyer.com/Es-Herb/Barberry.htm, acessada em junho de 2005.

Leite F 1989. Validação em Análise Química. 3 Ed. São Paulo: Editora Átomo.

Martins ELP, Brandão MGL 2006. Qualidade de amostras comerciais preparadas com Aesculus hippocastanum L. (Castanha-da-Índia). Rev Bras Farmacogn 16: 224-229.

PDR for Herbal Medicines 2000. Montvale, New Jersey: Medical Economics Company.

Pimentel MF, Barros NB 1996. Calibração: uma revisão para químicos analíticos. Quim Nova 19: 268-277.

Pizzorno JE, Murray MT 1985. A Textbook of Natural Medicine. Seattle, Washington: John Bastyr College Publications.

Preininger V 1975. The Pharmacology and Toxicology of the Papaveraceae alkaloids. In: Manske RHF, Holmes
HL. The alkaloids. V. 15. New York: Academic Press, p. 239.

Ribani M, Bottoli CBG, Collins CH, Jardim ICSF 2004. Validação em métodos cromatográficos e eletroforéticos. Quim Nova 27: 771-780.

Ribeiro AQ, Leite JPV, Dantas-Barros AM 2005. Perfil de utilização de fitoterápicos em farmácias comunitárias de Belo Horizonte sob a influência da legislação nacional. Rev Bras Farmacogn 15: 65-70.

Sreevidya N, Mehrotra S 2003. Spectrophotometric method for estimation of alkaloids precipitable with Dragendorff's reagent in plant materials. $J A O A C$ Int 86: 1124-1127.

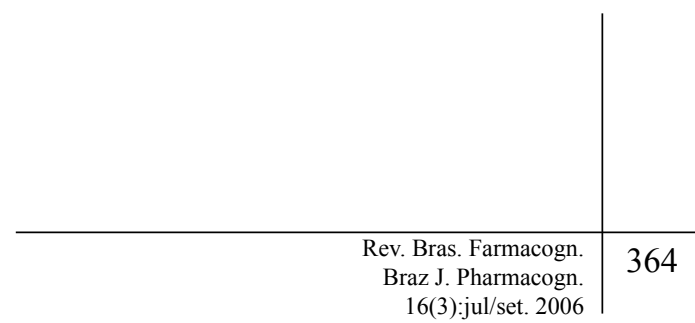

